\title{
A SHORT REVIEW ON METRIC SPACE
}

\author{
MANISHA KAISTHA and MEENAKSHI \\ E-mail Id:20MSM1019@cuchd.in, chawlameenakshi7@gmail.com \\ Department of Mathematics, UIS \\ Chandigarh University, Mohali, Punjab, India
}

\begin{abstract}
This section is devoted to the study of the measuring area in which topology set $\mathrm{X}$ is produced by an unused scalar known as metric that can be defined as measurement of a particular distance between any two parts, or focused, in the setting as part of its properties strongly suggests the natural concept of distance we usually experience. In everyday life. This type of climatic space includes a popular position among all technological spaces because the reason is that its topology has been completely removed by scale-level work. We can confidently assume that we are well acquainted with the characteristics of such work and are accustomed to handling it. All things being equal, perfect topology is often commended by a certain category of a small dynamic subset of a theoretical set. The concept of measurement space was first introduced by Fréchet in year 1906. However, the name of the metric space was given by Hausdorff later.
\end{abstract}

\section{KEYWORDS}

Cauchy sequence, open ball, Topological space, Equivalent class, Contraction mapping

\section{INTRODUCTION}

This section is provided in an exploration of the measurement space in which the topology in set $\mathrm{X}$ is created by a scalar function with a false value called metric which can be defined as measuring a certain distance between any two parts, or focused, of setting as part of its structures strongly much in everyday life. This type of geography includes or perhaps a beneficial position in all the topological spaces because its topology has been completely removed by scalar distance work. We can confidently imagine that we are well acquainted with the characteristics of such work and are accustomed to controlling it. All things considered; complete topology is a rule approved by a particular category of very small subset of theoretical set. The concept of measurement space was first introduced by Frechet in the year 1906. However, the name of the metric space was coined by Hausdorff later. [1] 
In this section we start by placement of the scalar function structures in set $\mathrm{X}$ so that that is known as the scale. At that point we are talking about how we can create the cause of a particular topology in $\mathrm{X}$ by abusing its function properties. Next we will move further with the consideration of a few models that have different values and are widely used in various applications. At that time a number of spatial structures, for example, functional integration, sequence integration, and set size and so on, will be considered from a metric point. At that time it is very similar to the geology of the earth, and as another concept, the metrics of the metrics of the metric spaces will be analysed. The theme of fulfilment, identified by a mix of Cauchy arrangements in the rating spaces, and Baire's planning will be re-examined. Then we will provide a useful strategy for obtaining the climax of a divided space. Following the management of the issue map and its focus areas, we will discuss the reduction of metric gaps and specific conservation measures arising from the requirements of the metric metric. At that point the pen will finally talk about the matrix structures of the Functional Analysis set that have a real number of ongoing functions in the measurement spaces and the significance of the measurement hypothesis. Finally we will present an integrated discussion of fractal structures within the matrix space system. [2]

\section{BACKGROUND OF METRIC SPACES}

There are collection of ways to deal with evaluate the distance between two concentrations or two vectors. While the theory of estimation past the Euclidean spaces was started by the Italian mathematicians like Ascoli or Volterra, it at first got an understandable and theoretical assessment in Fréchet's doctoral proposition of 1906 where the normal considerations of Euclidean space past the examination of mathematical figures were embraced. The hypothetical spaces coming about in view of such an assessment were portrayed by their particular segments, adage, and their mix. Also, limits was applied by Fréchet from math in viewing the limits as parts of a vector space, which was in like manner a technique for assessing lengths and distances amidst the abilities to convey a measurement space. He recognized two basic kinds of summarized spaces: The L-spaces, where the possibility of limit relied upon the platitude of simultaneous game plans, and the second being the L-spaces where a distance limit can be described. Assessment was continued by various mathematicians for instance Finsler, Hausdorf or Wilson and that finally incited the blend of the properties of metric spaces.[3]

In number juggling, a measurement space is a set where a distance (called a measurement) is portrayed between components of the set. Metric space strategies have been used for a significant long time in various applications, for example in web search apparatuses, picture gathering, or protein game plan. In oil supply displaying, metric space procedures are not by and large known, and few uses of metric space systems have been presented. The slow gathering is likely a result of the way that a colossal a lot of repository demonstrating inspects are performed using a solitary supply model which is adjusted to unquestionable data and a short time later used for examination and dynamic. 


\section{THE METRIC AND THE METRIC TOPOLOGY}

Consider a set $X$ and a function $d: X-\rightarrow R$, a pair $M=(X, d)$ is a metric space iff $d($.$) satisfies$ the following properties:

1) For all $x, y$ belongs to $X, d(x, y) \geq 0$ (Non-Negativity)

2) For all $x, y$ belongs to $X$ we have, $d(x, y)=0=>x=y$ (Identification)

3) For all $x$, $y$ belongs to $X, d(x, y)=d(y, x)$ (Symmetry)

4) For all $x, y, z$ belongs to $X$, we have that: (Triangular inequality) $d(x, z) \leq d(x$ ,y) $+\mathrm{d}(\mathrm{y}, \mathrm{z})$

The function we call is ( $\mathrm{x}, \mathrm{y})$ metric in set $\mathrm{X}$ and defines the distance between objects $\mathrm{x}$ and $\mathrm{y}$ as its value of $\mathrm{X}$ which will therefore be referred to as the purposes of set $\mathrm{X}$. Thereafter, the scale function $\mathrm{d}(\mathrm{x}, \mathrm{y})$ in some cases is known as function distance to $\mathrm{X}$. Without a doubt, as our daily knowledge of the well-known concept of distance tells us, distance must remain positive and disappearing if the two set objectives are equal, the distance between the two focuses is equal from any measured point in terms of the fact that metric is the equal function of its arguments. The final asset indicates that in the case of a three-dimensional design of structures, then the total length of the different sides of the triangle may not be the length of the third dimension. In the event that we release the second axiom better than $\mathrm{x}=\mathrm{y}$ suggest and $d(x, y)=0$ yet it is currently allowed that $d(x, y)=0$ does not expect ix to be equal to $y$, then the most prohibitive function $d$ is known as pseudo-metric or ecart. [4]

An open sphere with radius $r>0$ centered at $\mathrm{x}$ where $\mathrm{x}$ belongs to $\mathrm{X}$ is defined as following subset of X:

$\mathrm{B}_{\mathrm{r}}(\mathrm{x})=\{\mathrm{y}$ belongs to $\mathrm{X}: \mathrm{d}(\mathrm{x}, \mathrm{y})<\mathrm{r}\} \mathrm{C} \mathrm{X}$

If we consider $r=0$,

there is no purposes of $\mathrm{X}$ having a place with the set $\mathrm{B}_{\mathrm{o}}(\mathrm{x})$ since the metric can't be negative, specifically, we get $\operatorname{Bo}(x)=0$. Let us now consider a class of subsets $\operatorname{Il} 3 d=\{\operatorname{Br}(x): x E X, r$ $2:: \mathrm{O}\}$ of the set $\mathrm{X}$. It is evident that $\mathrm{X}=\mathrm{U}\{\mathrm{Br}(\mathrm{x}): \mathrm{x} \mathrm{E} \mathrm{X}, \mathrm{r}>\mathrm{O}\}$. T

\section{APPLICATIONS OF METRIC SPACE}

\section{APPLICATIONS OF METRIC SPACES IN QUANTUM MECHANICS:}

Hilbert's space includes buildings of two different types of price spaces: vector space and metric space. While vector-space angles are widely used, standard viewing areas are used the most. We show that the laws of quantum mechanical preservation often lead to the matrix spaces for the arrangement of the related body mass1. All such measurement spaces have 
"onion-shell" figures. The connected scale divides the Fock space into concentrated sectors where large and small distances between countries can be identified and mathematically divided. In contrast to the typical Hilbert area survey, our results work similarly in the narrower space of the ground and in the cellular density, which is metric, but not Hilbert, spaces. The Hohenberg-Kohn map between dense and terrestrial areas, which is particularly mind-boggling and non-local in facilitating visualization, is available, because the investigated frameworks are straightforward on a scale, when they turn into a monotonic map and almost directly to vicinities. Basically, by considering the measurement gaps associated with the many body components embedded in the magnetic field, we look at mapping between wave performance and (current strength and molecules) in the center of the Current Density functional hypothesis. We find, in the connected measurement spaces, the permissible and non-permissible range regions, the "band structure", which is directly related to the maintenance of the direct pressure component1. Finally, the latest results include appropriate estimates of external forces that allow us to investigate the " third leg " of the Hohenberg-Kohn theorem3.

\section{METRIC SPACE IN CONVERGENCE AND ITS APPLICATIONS:}

Metric space is the state where bunch blends can be seen: Let $\left(x_{n}\right) \mathrm{n} \in \mathrm{N}$ be gathering and when point in $\mathrm{M}$. We express that $\left(x_{n}\right) \mathrm{n} \in \mathrm{N}$ joins to $\mathrm{x}$, and make $\lim \mathrm{n} \rightarrow \infty x_{n}=\mathrm{x}$, if there is one number $\varepsilon>0$ there is an entire number $n_{0}$ with the ultimate objective that in every one of the entire numbers $\mathrm{n}>n_{0}$ the disparity $\mathrm{d}\left(x_{n}, \mathrm{x}\right)<\varepsilon$ is fixed. Individuals were educated about school abilities limits and utilized $\operatorname{limx} \rightarrow \infty \mathrm{f}(\mathrm{x})$. Our definition above is an alternate circumstance where the spot of $\mathrm{f}$ is a bunch of opposing qualities, i.e., $\mathrm{f}: \mathrm{N} \rightarrow \mathrm{R}, \mathrm{f}(\mathrm{n})=x_{n}$. This, in any case, has not been tried in schools. Likewise, as far as possible are not completely indicated. Regardless, we found that our more youthful pursuers had a characteristic impulse as far as the mark of the break point. We have utilized the application to pass on that the idea of metric space is at present thinking about a typical importance. This is a reasonable position picked contrasted with a solitary characteristic idea. Additionally, it shows that the relationship is anything but a critical factor of genuine numbers, yet relies upon the assurance of the metric.[6]

\section{CONCLUSION}

In this paper we discussed about metric spaces, definitions of metric spaces, theory and applications of metric spaces in quantum mechanics, in convergence and applications of metric spaces in daily life. Metric spaces is an important topic to be discussed in the stream of mathematics. We also discussed about background and discovery of metric spaces which will help us to understand their origin . In this paper we also discussed about topology and metric spaces. This gives us brief about metric spaces in almost every aspect.

Metric space techniques are best utilized to rapidly examine and decipher a gathering (troupe) of reservoir models and are along these lines appealing strategies for vulnerability studies or affectability examination when a (enormous) group of reservoir models is utilized.[5] 
A metric space (MS) in reservoir modeling is characterized by a uniqueness distance which estimates the divergence between pairs of reservoir models, shown schematically in the figure underneath (left). The distance measure applied over all model pairs shapes a distance grid appeared in the figure underneath (focus). The distance framework characterizes the metric space. The metric space can be imagined utilizing multi-dimensional scaling (MDS), whose yield is appeared in the figure beneath (right). MDS takes the distance network, and makes an interpretation of the models into focuses in an Euclidean space that can be pictured. The area of the focuses (models) in Euclidean space are enhanced to best surmised the distances somewhere far off framework. The MDS plot is a visual and demonstrative apparatus, permitting the representation of model vulnerability and reaction affectability. MDS is a dimensionality decrease strategy, like PCA.

\section{REFERENCES}

1. I.A. Rus, Generalized Contractions and Applications, Cluj University Press, ClujNapoca, Romania, 2001.

2. I. A. Rus, A. Petrus, el, and G. Petrus, el, Fixed Point Theory, Cluj University Press, Cluj-Napoca, Romania, 2008.

3. J. Caristi, "Fixed point theorems for mappings satisfying inwardness conditions," Transactions of the American Mathematical Society, vol. 215, pp. 241-251, 1976.

4. T. Abdeljawad and E. Karapinar, "Quasicone metric spaces and generalizations of Caristi Kirk’s theorem,” Fixed Point Theory and Applications, vol. 2009, Article ID 574387, 9 pages, 2009.

5. A. Amini-Harandi, "Some generalizations of Caristi's fixed point theorem with applications to the fixed point theory of weakly contractive set-valued maps and the minimization problem,” Nonlinear Analysis: Theory, Methods \& Applications, vol. 72, no. 12, pp. 4661-4665, 2010.

6. E. Karapinar, “Generalizations of Caristi Kirk’s theorem on partial metric spaces,” Fixed Point Theory and Applications, vol. 2011, article 4, 2011.

7. I. Kramosil and J. Michalek, "Fuzzy metrics and statistical ' metric spaces," Kybernetika, vol. 11, no. 5, pp. 336-344, 1975.

8. M. Grabiec, "Fixed points in fuzzy metric spaces,” Fuzzy Sets and Systems, vol. 27, no. 3, pp. 385-389, 1988. 